\title{
A Comparative Study of Emotion Metaphors between English and Chinese
}

\author{
Xiao Liu \\ Inner Mongolia University of Finance and Economics, Huhhot, China \\ Guodong Zhao \\ Inner Mongolia University of Finance and Economics, Huhhot, China
}

\begin{abstract}
Different from traditional linguistics which views metaphor as a means of rhetoric, cognitive linguistics holds that metaphor, instead of being a deviant phenomenon of normal language, is a way of thought and a powerful instrument of cognition. Because of the reason that the traditional linguistics failed to explain the essence of metaphor, the modern linguists began to do research on it from the perspective of language and philosophy. Metaphor has become an essential part in people's cognitive mechanism instead of being perceived as language phenomenon ever since the publication of masterpiece Metaphor We Live By contributed by Lakeoff and Johnson. In this thesis, cognitive linguistics approach is used to make a comparative analysis between the Chinese and English emotion conceptual metaphors. Conceptual metaphor, as an important concept in cognitive linguistics, refers to something internal, opening deep in human thought, such as ARGUMENT IS WAR and TIME IS MONEY. Emotions, as an important aspect of human experience, have been among the focuses of cognitive linguistics. Emotions are conceptualized and expressed in metaphorical terms. This thesis takes up emotion conceptual metaphors as its topic to study, trying to conduct a cross-linguistic research into the conceptualization of emotion concepts. Based on the analysis of linguistic data from English and Chinese, it tends to explore the similarities and differences between Chinese and English in terms of conceptual metaphors of the two most basic emotions including happiness and sadness.
\end{abstract}

Index Terms - conceptual metaphor, happiness, sadness, emotions, comparative analysis

\section{INTRODUCTION}

\section{A. Research Background}

Metaphor enjoys a long history in its evolvement from the early comparison, substitution and interactive theory to current conceptual metaphor, reflecting dramatic changes in people's view towards the relationship between language, mind and society. Aristotle, one of the earliest philosopher and typical representatives in metaphor studies, suggests that metaphor plays a deviant and aberrant rhetorical role and serve as an ornate and emotive instrument. So metaphor is nothing more than literal decoration aimed at impressing others. Accordingly, most metaphor studies in discourse analysis have been carried out by looking into language itself without taking into consideration social and ideological factors. However, by 1980s, George Lakeoff and Mark Johnson challenged all the powerful tradition theory in a systematic way in their ground breaking studies of Metaphor We Live By. Lakeoff and Johnson draw close attention to the relationship between language and thought and convincingly argue that metaphor is not simply a matter of linguistic expression but of concepts, of thinking of one thing in terms of another. What makes the contemporary theory of metaphor unique is the important distinction between conceptual metaphors or metaphorical concepts on the one hand, and linguistic metaphors or metaphorical expression on the other hand (Lakeoff, Johnson, 1980). The distinction between conceptual metaphor and linguistic metaphor is the major issue that distinguishes cognitive view of metaphor from the traditional view of metaphor. Conceptual metaphor is referred to as the fundamental cognitive agency in our conceptualizing of the world by cognitive linguists, such as George Lakeoff and Mark Johnson (1980).

According to the above cognitive linguists, metaphor pervade everywhere in the everyday life of all people, regardless of their race, social status, educational background, sex, context, etc. Entertaining TV shows, which form a natural part of people's daily life of our times, is no exception. For this reason, this study has chosen two entertainment TV shows as the object for investigation and analysis.

\section{B. Aim of the Study}

As mentioned above, cognitive linguists take metaphor as a reflection of human cognition. In view of the fact about the cognitive approach to metaphor and current situation of emotion concepts studies, this thesis is designed to conduct a comparative study of conceptual metaphors of emotion between Chinese and English. I set the present task in the hope of contributing my bit to the cognitive theory of metaphor, and also in a way, to cross-cultural communication as well.

\section{Research Methodology}

In this thesis, the research methodology is mainly qualitative. It takes a series of claims as its assumption. These 
claims include: human thought is basically metaphorical; our conceptual metaphor arises from our bodily experience; and the set-up conceptual metaphors impose the structure onto real life and influence our understanding and cognition (Lakeoff, Johnson, 1980).

\section{Source of Data}

The linguistic data, on which this study is based, are all derived from ordinary language used by and familiar to native speakers. The data are mainly taken from magazine, novel, short stories, folk/popular songs and everyday language talking about people's the most basic emotions of HAPPINESS and SADNESS.

\section{LITERATURE REVIEW}

\section{Conceptual Metaphor Theory}

The publication of Metaphor We Live By by the American cognitive linguists George Lakeoff and Mark Johnson in 1980 marked a turning point in metaphor studies since metaphor is regarded as a fundamental cognitive competence and is unconsciously and pervasively employed in people's daily life. As Lakeoff put it,

"Metaphor is pervasive in everyday life, not just in language but in thought and action. Our ordinary conceptual system, in terms of which we both think and act, is fundamentally metaphorical in nature." (George Lake off\& Mark Johnson, 1980)

In short, the locus of metaphor is not in language at all, but in the way we conceptualize one mental domain in terms of another, dispelling the traditional view that metaphor is the realm of poetic or figurative language. Then metaphor seems to be with us all the time whether one is aware of it or not. And as far as the nature of metaphor is concerned, something abstract is understood in terms of something more specific.

\section{Working Mechanism of Conceptual Metaphor}

When using a metaphor, like "Life is a journey", we are likely to assume that the speaker does not mean what he says literally but that he is speaking metaphorically. But how it is possible for speakers to communicate to hearers when they are speaking metaphorically in as much as they do not say what they mean, that is, how does metaphor work? This is an essential question for every metaphorical theory to answer.

There are two important concepts involved in metaphor, that is, the target domain - "the starting point", and the source domain - "the comparison point" (Saeed, 2000). And the emergence of metaphor is due to the comparison between the two domains. And the comparison is realized by a process of mapping.

The studies on metaphorical mappings have been made by many scholars, such as Lakeoff and Johnson (1980), Sweetser (1990), and Fauconnier (1997). The main idea of metaphor is expressing and understanding one kind of thing in terms of another, that is, metaphor can be understood as the mapping from a source domain to a target domain. Lakeoff just applies the term "mapping" to represent the interaction between two domains. Their working together provides us with the relationship between mapping and metaphor:

Each metaphor has a source domain, a target domain, and a source-to-target mapping;

Metaphor is the mapping from the source domain to the target domain;

Mapping is the systematic set of correspondences that exists between constituent elements of the source and the target domains;

The properties mapped from the source domain must fit the inherent structure of the target domain;

After mapping, the properties of source domain go with those of target domain.

To well understand mapping, we should know some traits of mappings for the sake of avoiding misunderstanding and obtaining a better comprehension of mappings.

First, metaphorical mappings obey the hypothesis called the Invariance Principle: the image schema structure of the source domain is projected into the target domain in a way that is consistent with inherent target domain structure. (Lakeoff, 1993)

Another principle is Target Domain Overrides, which decides what properties in the source domain can be mapped. Mappings are partial in that only a part of the properties and structures of the source domain are mapped onto the target domain.

The two principles explain a third characteristic of asymmetry of Mapping, that is, metaphors are directional. The transfer is from the source domain to the target domain.

\section{Classification of Conceptual Metaphor}

In Lakeoff and Johnson's point of view, conceptual metaphors can generally be divided into three main types: orientational metaphor, ontological metaphor and structural metaphor.

\subsection{Orientational Metaphor}

Orientational metaphors are also called spatialization since most of them result from our perception of space. Spatial orientations, derived from our constant interaction with our environment and experiences in the physical world, are the basic concepts by which we live, including up and down, in and out, front and back, deep and shallow, central and peripheral. These spatial orientations are directly grounded in the most basic experience of human beings and the experience in sensing orientations can be acquired in the early stage of human growth. This phenomenon has been proved to be correct in psychological studies. So it is natural that we use basic orientation concepts derived from these 
basic experience of this kind to understand more abstract concepts such as emotions, health conditions, quantity and social status, etc.

Orientational metaphors give a concept a spatial orientation, for example, in sentences "M spirit rose" and "I'm feeling down", the italic words rose and down are the application of orientational metaphors.

\subsection{Ontological Metaphor}

As human's primary way of existence is substantial, our experience of physical objects and substances allows us to understand the world and ourselves beyond mere orientation. Thanks to those experiences, we can pick out parts of those experiences and treat them as discrete entities or substances of a uniform kind. Just as the basic experiences of human spatial orientations promotes orientational metaphors, our experiences with physical objects (especially our own bodies) provide the basic for an extraordinary wide variety of ontological metaphors, that is, ways of viewing events, activities, emotions, ideas, etc., as entities and substances.

Ontological metaphor is generated when our experience with physical objects and substances make it possible to conceive of abstract intangible concepts such as emotions, ideas, psychological activities and states as concrete tangible entities and substances.

\subsection{Structural Metaphor}

In addition to orientational metaphor and ontological metaphor, structural metaphor is another important form of conceptual metaphor. When orientational metaphor and ontological metaphor are elaborated in much specific terms, or when some aspects of a concept are highlighted, we turn to structural metaphor for help. Structural metaphors allow us to used one highly structured and clearly delineated concept to structure another (Lakeoff \& Johnson, 1980). Structural metaphor is the case where one structured domain is metaphorically restructured and redefined in terms of another, that is to say, by virtue of the structure of a more familiar and concrete domain, we understand the abstract domain.

\section{Features of conceptual Metaphor}

Owing to the specialty of the relationship between metaphor and human experience, metaphor has its own features: the ubiquity, systematicity, asymmetry and abstraction.

The ubiquity of metaphor has been noticed by many researchers, like Gibbs (1994), Lakeoff \& Johnson(1980), and McNeil (1992). These researchers provide evidences for the prevalence of metaphor is not a privilege of a few specific groups but are ubiquitous throughout both written and spoken discourses.

The second feature, "systematicity, refers to the way that a metaphor does not set up a single point of comparison: features of the source and target domain are joined so that the metaphor may be extended, or have its own internal logic." (Saeed, 2000.) when pervading all kinds of discourses, these conceptual metaphors do not appear isolated from each other. On the contrary, they are closely related, and "metaphorical entailments can characterize a coherent system of metaphorical concepts and a corresponding coherent system of metaphorical expressions for these concepts' (Lakeoff \& Johnson, 1980). And consequently, a series of metaphors based on the same experience basis, or image schema, can run through and connect the whole discourse together, so that the reader can understand the discourse lightly.

The third one, asymmetry, according to Saeed, indicated the directional tendency of metaphors. The comparison between the two concepts, or domains, is not symmetric, on the contrary, they only "transfer features from the source to the target" Saeed, 2000).

The final one, abstraction, is related to the asymmetry, "It has often been noted that a typical metaphor uses a more concrete source to describe a more abstract target." (Saeed, 2000) Meanwhile, Saeed also points out that this is not a necessary feature of metaphors: the source and target domains may be equally concrete or abstract.

\section{Significance of conceptual Metaphor}

As Lakeoff indicated, compared with the traditional theories of metaphor, the conceptual metaphor theory was revolutionary in many respects (Lakeoff, 1993). Metaphor was no longer viewed as a figure of speech, to the "novel or poetic linguistic expression" (ibid). Instead it is a way of thinking, and everyday abstract concepts like time, states, change, causation, and purpose also turn out to be metaphorical" (ibid).

After the publication of Metaphor We Live By, the landmark of cognitive study of metaphor, more and more scholars devoted themselves to this field and other applied disciplines, Henderson (1982) examined metaphor in economics texts; Johnson (1987) argued for the "bodily basis of meaning, imagination, and reason'; Lakeoff and Turner(1989) showed the relevance of the ordinary language metaphoric system for the interpretation of literature; Lakeoff (1991) analyzed the metaphoric system that served to justify war in the Gulf; Fesmire (1994) investigated the body metaphors used to structure mental functioning; Lakeoff (1993) studied the applicability of conceptual metaphor to dream analysis. Steen's remark is quite succinct: "This cognitive approach to metaphor has grown into one of the most exciting fields of research in the social sciences, with psychologists leading the way for cognitive linguists, anthropologists, and poeticians" Steen, (1994)

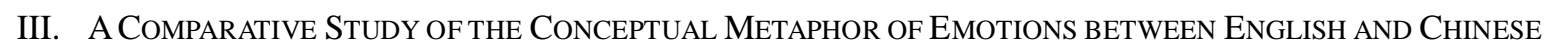

Metaphor is a means of conceptualizing human being's bodily experience and understanding the world, through which we comprehend abstract concepts and perform abstract reasoning. Since cognition can influence and be influenced by emotions, the study of human emotions plays an important role in the probe into human cognition. Hu 
Zhuanglin (2004) once said that language is regarded as an important tool in the job of learning about cognition and the world. Language of emotions is an effective access to the adequate study of emotions, and the linguistic analysis has been an essential source of information about emotion concepts. In the following part, what will be discussed are the conceptual metaphors of the two basic emotions: happiness and sadness between English and Chinese.

\section{A. HAPPINESS Metaphors between English and Chinese}

\section{Similarities}

In English, according to Lakeoff and Johnson (1980), a major conceptual metaphor of happiness is an orientational one: HAPPINESS IS UP. Under this conceptual metaphor, there are some expressions:

a. I'm feeling up.

b. He is in high spirit.

c. We try to keep her spirits up.

d. Cheer up, boy

e. Come on!

In Chinese, there are also similar expressions:

Ta de yi yang yang.

Wo men qing xu gao zhang.

Yi xia zi ti qi le wo de xing zhi.

Ta zheng zai xing tou shang.

Wo hen xing fen.

These expressions reveal that happiness is conceptualized in terms of an upward orientation. Actually most of them are so conventionalized that they may not be taken as metaphorical concepts by native speakers. These expressions are in effect grounded in the human's bodily experience. They arise from the fact that we have upright bodies and the effect posture typically goes with positive valuation of emotional states as well as physical states.

In addition, English and Chinese both have the conceptual metaphor HAPPINESS IS FLUID IN CONTANER, which maps the emotion of happiness onto a fluid in a container. This conceptual metaphor in fact is a specific-level instantiation of the more general metaphor THE EMOTIONS ARE IN A CONTAINER. It is manifested in English by the examples below:

My heart is full of joy

He is bursting with joy.

I was overwhelming with joy.

Joy welled up inside her.

The conventionalized linguistic expressions under this conceptual metaphor in Chinese include:

Ta xin zhong cong man le xi yue.

Ta men man huai xi yue.

Ta dui sheng huo cong man le zhi re de qing gan.

Yi zhong huan yu yong shang le xin tou.

Kovecses (1986) states that the body is viewed as a container, the emotions are conceived of as the fluid inside a closed container. When emotions are strong enough and beyond the limitation of the container, it will overflow, burst, so we have the linguistic expressions mentioned above. Although English and Chinese share the CONTAINER metaphor in expressing happiness, there are descriptive differences between them according to the examples without using "heart" are very common; Chinese tends to use more body parts, such as heart, bosom or chest as the container of happiness.

\section{Differences}

There are some differences in the conceptual metaphor of happiness between English and Chinese. Through careful comparison and analysis, the following major differences concerning HAPPINESS metaphors can be observed. Here, I take the metaphors HAPPINESS IS BEING OFF THE GROUND \& HAPPINESS IS FLOWERS IN THE HEART as an example:

In English, besides the above-mentioned conceptual metaphors depicting happiness, there is another conceptual metaphor of upward orientation: HAPPINESS IS BEING OFF THE GROUND. Instances of this type include:

They were in the clouds.

He was just soaring with happiness.

I'm six feet off the ground.

I was floating.

As is observed, this metaphor may be partially concerned with birds, which are usually viewed as symbol of freedom, and thus associated with happiness.

While in Chinese, the HAPPINESS IS BEING OFF THE GROUND metaphor does not seem to be applicable due to our Chinese conventional mode of thought. Chinese people regard "being off the ground" as the indication of being proud, conceited and out of control. When we say "ta you dian piao piao ran le" and "ta you zai yun li wu li le", we mean that the person is so smug that he has lost his sense. Instead, we have modesty and prudence as virtues by 
adhering to the sayings, "de yi bu neng wang xing" and "jiao tra shi di".

Instead of being described as BEING OFF THE GROUND, happiness in Chinese is sometimes depicted in terms of FLOWERS IN THE HEART. For instance:

Da he chang na le yi deng jiang, wo men yi ge ge xin huan nu fang

Shou dao lao shi de biao yang, ta xin li le kai le hua.

This metaphorical concept, though a minor one, is rooted in the Chinese culture, in which flowers, especially "big red flowers" are traditionally considered as symbol of happiness. Meanwhile, this FLOWER metaphor somehow mirrors the introverted character typical of Chinese people: reaction to happiness in the heart is highlighted. This forms a sharp contrast with westerners who are more extroverted and tend to express emotions more outwardly, just as the metaphor HAPPINESS IS BEING OFF THE GROUND reflects.

\section{Summary}

To summarize, English and Chinese share some major conceptual metaphors in conceptualizing happiness, which include: HAPPINESS IS UP, HAPPINESS IS FLUID IN A CONTAINER. However, some differences exist in the conceptual metaphor of happiness between English and Chinese, as illustrated by HAPPINESS IS BEING OFF THE GROUND from English, HAPPINESS IS FLOWERS IN THE HEART from Chinese and Chinese tends to use more body-part nouns in the expressions of happiness, such as performing the bro together with eyes to show the emotion of happiness.

\section{B. SADNESS Metaphors between English and Chinese}

\section{Similarities}

Sadness is an emotion opposed to happiness. In most circumstances, a contrast exists between the conceptualization of the two emotions, which means that sadness has the similar source domains to happiness in some aspects. The first major conceptual metaphor characterizing the conceptualization of sadness in English is an orientational one: SADNESS IS DOWN. Under this conceptual metaphor, there are many conventional expressions:

I'm feeling down.

He's really low these days.

$\mathrm{He}$ is in low spirits.

His spirits fell at the bad news.

I'm depressed.

These examples use words like down, low, fall and depressed to express the emotion of sadness. It is not randomly to apply such spatial words to indicate emotion, but a close relation to our bodily experience. As human beings have upright bodies, our postures are mapped to the understanding of emotions. The erect posture typically goes with positive emotions as well as physical states, such as happiness and health; on the contrary, the negative emotions such as sadness and illness often accompany a drooping posture. In the process of human cognition, spatial concepts are projected onto emotional concepts on the basis of the relevance of human posture and emotions, thus comes the result--- the concept of happiness is expressed with up-words and phrases, while the concept of sadness is expressed with down-words and phrases.

The conceptual metaphor SADNESS IS DOWN is also found in Chinese, which is manifested by extensive expressions. For example:

Zhe ji tian ta qing xu hen di luo.

ta hen xiao chen.

Ta zong shi yi fu cui tou shang qi de yang zi.

Wo zong shi da bu qi jing shen lai.

Zhe xiao xi jian zhi rang ta de xin die dai di gu.

Obviously, Chinese has the similar uses in conducting DOWN to map onto SADNESS.

In addition, SADNESS is ordinary conceptualized as dark. The use of this metaphor SADNEDD IS DARK is similar in English and Chinese. The linguistic realization of this metaphor is illustrated below:

He is in a dark mood.

The future looks pretty dismal

Both of us were in a black mood.

I felt very gloomy.

In Chinese, SADNESS IS DARK metaphors are easily found. For instance:

Ting dao zhe ge xiao xi, ta yan qian yi zhen hei, yun dao guo qu.

Ta lian shang hao xiang tu ran meng shang le yi ceng wu yun.

Hei se liu yue zhong yu guo qu le.

Ta mu guang an dan.

In the examples listed above, it presents a figure with the emotion of sadness in the cold, dark color through the words such as dark, gloom, dismal and hei se, an dan, wu yun etc. These expressions are aroused by our experience. It is observed by human beings that when a person, either an English people or a Chinese, becomes sad, he or she feels a loss of control over the situation and perceives his/her contribution purposelessly; and commonly, he or she has poor 
appetite and loss vigor; his/her complexion and eyes turn darker. The emotion of sadness then is linked with darkness. And projection from the lightness domain onto the emotion domain is established in human cognition and languages.

\section{Differences}

English and Chinese belong to two different linguistic systems and have one's own cultural custom, which have the influence on the conceptualization of the things around them. In the metaphorical concept of sadness, differences appear in the two languages.

English has the conceptual metaphor SADNESS IS BLUE but it does not exist in Chinese. Underlying this metaphor, there are many expressions:

She looks blue today.

Rainy days make me blue.

Paul was in a blue mood.

It was blue Monday and he didn't feel like going back to work.

Similarly, there also exists a sadness metaphor peculiar to Chinese. Chinese employs another commonplace conceptual metaphor, which is not so obvious in English, namely SADNESS IS PAIN IN HEART, from which many conventional expressions are derived:

E hao chuan lai, ta de xin dun shi shen shen di chi tong.

Xin tong de gan jue, jiu xin che fei.

Ta mo mo cheng shou zhe wan jian chuan xin de tong $k u$.

Ta xin ru dao jiao.

Besides "heart", Chinese people tend to use "intestines" to depict sadness, as shown in such phrases as:

gan chang cun duan.

duan chang ren zai tian ya.

chou chang cun duan.

This metaphorical concept to a certain extent mirrors our Chinese character which is characterized by introversion and pessimism, compared with extroversion and optimism embodied in western people's character. When Chinese people encounter something unhappy, for most of them, the actions they perform are to keep it in heart, for in Chinese traditional culture, it advocates an idea of "ren", they can seldom take them slightly. Sadness is often hidden inside, which causes pain in the heart.

3. Summary

To sum up, through the above examples and illustrations, it shows that in conceptualizing the emotion of sadness, English and Chinese share many similarities in metaphors, such as SADNESS IS DOWN and SADNESS IS DARK. Differences exist in some aspects because of cultural influences. English has the metaphor SADNESS IS BLUE, while SADNESS IS PAIN IN HEART belongs to Chinese. In addition, Chinese tends to utilize more body parts in the expressions for sadness than English does.

\section{CAuses For the Similarities AND DifFERENCES of THE CONCEPTUAL METAPHOR OF EMOTIONS IN ENGLiSH AND CHINESE}

The previous chapter has presented a comparative study of conceptual metaphors of the two basic emotions: happiness and sadness between English and Chinese. As the study reveals, English and Chinese share some major conceptual metaphors in the conceptualization of the two emotions, but they differ from each other in other aspects.

\section{A. Causes for Similarities: Shared Experience}

Language roots in people's cognitive structure and people talk about things the way they conceive of them. Richards (1936) states "Language is the joint point of different experience in different fields. It is not only the manifestation of cognition, but also part of it, the cognitive system coming from daily experience constitutes the psychological basis of language performance". Lakeoff and Johnson (1980) also point out that systematic analysis of language use can indicate the presence of an underlying metaphoric conceptual process which can explain whole collections of expressions. From the comparative study on the emotion metaphors in English and Chinese in the former chapter, results illustrates that emotion concepts both in English and Chinese are largely conceptualized through the metaphorical process that goes from the more concrete domain to the more abstract domain. Besides this, it is found that the two basic emotions in the two languages are mostly in the major conceptual metaphors. English and Chinese belong to different languages and cultural system, how can they have so many similarities in the emotional conceptual metaphors? The reason is that the similarity is the product of human conceptualization which is profoundly influenced by certain universal properties of the human body. The properties here refer to the embodiment of meaning---meaning reflects "our collective biological capacities and our physical and social experiences as beings functioning in our environment" (Lakeoff, 1987). The fact that these capacities and experiences are same or similar in fundamental respects for human being accounts for the similarity in the expression of emotion between English and Chinese. English-speaking and Chinese-speaking people appear to have very similar ideas about their bodies and seem to see themselves as undergoing the same or similar physiological and behavioral process in many cases when one person is in the state of happiness and sadness. All the similarities support that metaphors are not arbitrary; they are conceptual in nature and are based on human beings' 
bodily experiences.

\section{B. Causes for Differences: Cultural Relativity}

As pointed out in the previous chapter, there are differences of emotion conceptual metaphor existing in English and Chinese. The reason for the differences comes from cultural influence. Culture is the total pattern of beliefs, customs, institutions, objects and techniques that characterize the life of a human community. Language is a part of culture and plays a very important role in it, it is a social phenomenon and each language stands for one culture, so does in metaphor. Lakeoff (1980) also proposes that the most fundamental values in a culture will be coherent with the metaphorical structure of the most fundamental concepts in the culture.

English and Chinese belong to totally different language system: one of Indo-European, the other of Sino-Tibetan. English culture is greatly affected by the four-humor doctrine which originates from the ancient Western medicine and philosophy, while Chinese culture is greatly affected by the ancient Chinese philosophy and theories in Traditional Chinese Medicine. Different cultural influence brings out the differences in the conceptualization of the two emotions in English and Chinese.

\section{CONCLusion}

The thesis conducts a comparative study of the conceptualizations of emotion metaphors on happiness and sadness in English and Chinese. Conceptual metaphor means one conceptual domain is understood in terms of another conceptual domain, which is a projection of property and structure between the two domains.

The comparative analysis of the conceptualization of the two basic emotions in this thesis shows that English and Chinese share similarity in some major conceptual metaphors. The reason for these similarities between the two languages in the conceptualization of emotions are emotional concepts which are embodied, namely they have a basis in human being's bodily experience or they are closely related to the physiological functioning of human body. The similarities prove that metaphors are not arbitrary but embodied or motivated by the physiological reality.

Differences also exist in the conceptualizations of the two emotions between English and Chinese, which is due to the influence of concrete historical and traditional culture factors. Culture influences people's cognition of the world and concepts. In English cultural model, it holds the old west humoural doctrine, but principles in Chinese philosophy and the theories in Traditional Chinese Medicine play an important role in Chinese culture.

In short, conceptual metaphors of emotions are primarily rooted in bodily experience, but meanwhile, they are also influenced by cultural models. To put it in another way, emotion metaphors are characterized by cognitive commonness and cultural diversity. The findings of the study are also expected to help facilitate cross-cultural communication to certain extent by shedding light on the closely related intrinsic relations between language, mind and culture.

\section{REFERENCES}

[1] Hu Zhuanglin, (2004). Metaphor and Cognation. Beijing: Beijing University Press.

[2] John I. Saeed. (2000). Semantics. Beijing: Foreign Language Teaching and Research Press.

[3] Koveses, Zoltan. (1986). Metaphor of Anger, Pride, and Love: a Lexical Approach to the Structure of Concepts. Amsterdam: John Benjamins.

[4] Koveses, Zoltan, (1990). Emotion Concepts. New York: Springer- Verlag.

[5] Lakeoff, G. \& Johnson, M. (1980). Metaphors We Live by. Chicago: University of Chicago Press.

[6] Lakoff, G. (1993). The Contemporary Theory of Metaphor. A. Ortony (ed.) Metaphor and thought (2nd edition) Cambridge: CUP. 202-251.

[7] Gibbs, R. W. (1994). The poetics of mind: figurative thought, language, and understanding. New York, NY: Cambridge University Press.

[8] Lynda D. McNeil, (1992). Recreating the World. New York: State University of New York Press.

[9] Henderson, W. (1982). Metaphor in economics. Economics, 18(4), 147-153.

[10] Lynne Cameron \& Graham Low. (2001). Researching and Applying Metaphor. Shanghai: Shanghai Foreign Language Research Press.

[11] Lakoff \& Turner, (1989). More Than Cool Reason. Chicago: University of Chicago Press.

[12] Johnson. Mark, (1987). The Body in the Mind: The Bodily Basis of Meaning, Imagination and Reason. Chicago: University of Chicago Press.

[13] Fesmire, S. A, (1994). Aerating the Mind: The Metaphor of Mental Functioning As Bodily Functioning, Metaphor and Symbolic Activity 9, 31-44.

[14] Steen, Gerard. (1994). Understanding Metaphor in Literature: an empirical approach. New York: Longman Publishing, p263

[15] Kovecses, Z. (1986). Metaphors of Anger, Price and Love. A Lexical Approach to the Structure of Concept. Amsterdam and Philadelphia: John Benjamin's Publishing Company.

[16] Shu Dingfang. (2000). A Study of Metaphor. Shanghai: Shanghai Educational press.

[17] Wang Yin. (2007). Cognitive Linguistics. Shanghai: Foreign Language Education Press Shanghai.

[18] Eve Sweetser. (1990). From Etymology to Pragmatics: Metaphorical and Cultural Aspects of Semantic Structure: New York: Cambridge University Press.

[19] Fauconnier. G. (1997). Mapping in Thought and Language. Cambridge: CUP

[20] Richards I. A. (1936). The philosophy of rhetoric. Oxford Press. 
Xiao Liu was born in Baotou, inner Mongolia, in1976. She got Master's degree of Art at Foreign Languages College, Inner Mongolia University, Huhhot, in 2008.

She is working as a lecturer in Foreign Languages department of Inner Mongolia Finance and Economics college, Huhhot, P.R. China, teaching English Literature.

Guodong Zhao was born in Feng Zhen, Inner Mongolia, in 1975. He got Master's degree of Art at Foreign Languages College, Inner Mongolia University, Huhhot, in 2008.

$\mathrm{He}$ is working as an Associate professor in Foreign Languages department of Inner Mongolia Finance and Economics college, Huhhot, P.R. China, teaching Linguistics. 